\title{
A System of Comprehensive Technical and Economic Evaluation of Standardized Design Documentation
}

\author{
Olga Didkovskaya ${ }^{1}$, Aleksey Bocharov $^{1, *}$, and Tatyana Gordeeva ${ }^{1}$ \\ ${ }^{1}$ Samara State Technical University, Molodogvardeyskaya St., Samara, 443001, Russia
}

\begin{abstract}
One of the most important tasks of Russia's Ministry of Construction is the compilation of the registry of building designs approved for repeat use in public facilities construction. When completed, this registry will facilitate the design process and reduce building-related costs and construction timeframes. The existing guidelines, standards and regulations applicable to repeat designs are analyzed. Challenges hampering the broader use of repeat designs are assessed and possible solutions are suggested. The article presents a set of criteria for the selection of the most efficient designs recommended for repeat use (technical and technological evaluation, investment attractiveness, operation and maintenance costs estimate).
\end{abstract}

\section{Introduction}

One of the ways to encourage permanent construction of public facilities is the use of standardized designs (designs approved for repeat use, repeat designs). Russia's Ministry of Construction, Housing and Utilities is currently working on the Registry of Standardized Designs. So far, more than 200 designs have been added to the Registry. The following types of facilities are represented: residential, administrative, educational, healthcare, sports facilities and venues, cultural facilities, miscellaneous [8].

\section{Comprehensive Technical and Economic Evaluation of Standardized Designs}

To be added to the Registry, the design must fulfill the following requirement:

- The structure and the contents of the design documentation must be in compliance with the Russian legislation.

- The design must have a state expertise approval issued no more than 7 years ago;

- The design documentation must be in compliance with by-law land use and development regulations for the site where the design is to be repeated (including dimensional limits for permanent construction, e.g. the height of the building).

*Corresponding author: kafedra_cen@mail.ru 
- Its repeat use must be in compliance with the intellectual property rights in accordance with the civil legislation of the Russian Federation, i.e. if the design is to be repeated by anyone other than the owner of the rights, there must be a legal basis for it (e.g. a license agreement, a contract of uncompensated use etc.).

- The repetition on a new site must be possible and appropriate with regard to topography [2.8].

- Such designs must have the best performance in terms of:

- Building-related costs and service life estimate;

- The ratio of net floor area to gross floor area;

- Energy performance (resources consumption per unit of power necessary for the operation of the building);

- Innovative architectural, engineering, technical, technological and/or organizational solutions.

All designs added to the Register must have a portfolio containing sufficient information to help investors and developers get a full picture of the design and its main technical and economic parameters $[1,8]$.

However, the analysis of the design portfolios published by the Ministry of Construction raises some questions and ambiguities that need to be addressed. Table 1 shows part of the Registry.

Table 1. Registry of Standardized Designs.

\begin{tabular}{|c|c|c|}
\hline $\begin{array}{l}\text { No (type, } \\
\text { section, } \\
\text { design no) }\end{array}$ & Section & Design \\
\hline 1 & 2 & 3 \\
\hline 1.1 & \multicolumn{2}{|c|}{ Section 1. Low-rise residential buildings (manors) and townhouses- } \\
\hline 1.2 .1 & $\begin{array}{l}\text { Section } 2 \text {. Mid-rise residential } \\
\text { buildings (3-5 storeys) }\end{array}$ & $\begin{array}{l}\text { A 24-flat residential building for orphans } \\
\text { in the town of Nikolaevsk, Volgograd } \\
\text { region }\end{array}$ \\
\hline 1.2 .2 & $\begin{array}{l}\text { Section } 2 \text {. Mid-rise residential } \\
\text { buildings (3-5 storeys) }\end{array}$ & $\begin{array}{l}\text { A 24-flat residential building in Iglino, } \\
\text { Iglinsky administrative and municipal } \\
\text { district, Republic of Bashkortostan }\end{array}$ \\
\hline 1.2 .3 & $\begin{array}{l}\text { Section } 2 \text {. Mid-rise residential } \\
\text { buildings (3-5 storeys) }\end{array}$ & $\begin{array}{l}\text { A residential building for the disabled, } \\
\text { orphans and families with disabled } \\
\text { children in the city of Khabarovsk }\end{array}$ \\
\hline 1.3 .1 & $\begin{array}{l}\text { Section } 3 \text {. Multi-storey residential } \\
\text { buildings (6-10 storeys) }\end{array}$ & $\begin{array}{l}\text { A residential building at } 15 \text { a Montclair } \\
\text { St, 112th district of the city of } \\
\text { Cherepovets, Vologda region }\end{array}$ \\
\hline 1.3 .2 & $\begin{array}{l}\text { Section 3. Multi-storey residential } \\
\text { buildings (6-10 storeys) }\end{array}$ & $\begin{array}{c}\text { A 108-flat residential building in } \\
\text { Levitana St }\end{array}$ \\
\hline 1.3 .3 & $\begin{array}{l}\text { Section 3. Multi-storey residential } \\
\text { buildings (6-10 storeys) }\end{array}$ & $\begin{array}{l}\text { An apartment building at } 1 \text { Vishnevaya } \\
\text { St, in the Central district of Sochi }\end{array}$ \\
\hline 1.3 .4 & $\begin{array}{l}\text { Section 3. Multi-storey residential } \\
\text { buildings (6-10 storeys) }\end{array}$ & $\begin{array}{l}\text { An apartment building at } 2 \text { Vishnevaya } \\
\text { St, in the Central district of Sochi }\end{array}$ \\
\hline 1.3 .5 & $\begin{array}{l}\text { Section 3. Multi-storey residential } \\
\text { buildings (6-10 storeys) }\end{array}$ & $\begin{array}{l}\text { A residential building in Kirpichnaya St, } \\
\text { in the city of Murmansk }\end{array}$ \\
\hline 1.4 .1 & $\begin{array}{l}\text { Section } 4 . \text { High-rise residential } \\
\text { buildings (11-16 storeys) }\end{array}$ & An 88 -flat residential building \\
\hline 1.4 .2 & $\begin{array}{l}\text { Section } 4 \text {. High-rise residential } \\
\text { buildings (11-16 storeys) }\end{array}$ & $\begin{array}{l}\text { A residential building for the artistic } \\
\text { personnel of regional theatres and other } \\
\text { entertainment establishments in the city } \\
\text { of Krasnoyarsk }\end{array}$ \\
\hline
\end{tabular}




\begin{tabular}{|c|c|c|}
\hline 1.5 .1 & $\begin{array}{c}\text { Section 5. High-rise residential } \\
\text { buildings ( }>16 \text { storeys) }\end{array}$ & $\begin{array}{c}\text { A 17-storey large panel residential } \\
\text { building, BS1-R type (a 84-flat central } \\
\text { section with a 1-1-1-3-3 floor plan) }\end{array}$ \\
\hline 1.5 .2 & $\begin{array}{c}\text { Section 5. High-rise residential } \\
\text { buildings ( }>16 \text { storeys) }\end{array}$ & $\begin{array}{c}\text { A 17-storey large panel residential } \\
\text { building, BS2-R type (a 84- flat central } \\
\text { section with a 1-2-2-3 floor plan) }\end{array}$ \\
\hline 1.5 .3 & $\begin{array}{c}\text { Section 5. High-rise residential } \\
\text { buildings ( }>16 \text { storeys) }\end{array}$ & $\begin{array}{c}\text { A 17-storey large panel residential } \\
\text { building, BS3-Tl type 1 (a 67-flat left- } \\
\text { end section with a 2-2-2-2 floor plan) }\end{array}$ \\
\hline 1.5 .4 & $\begin{array}{c}\text { Section 5. High-rise residential } \\
\text { buildings ( }>16 \text { storeys) }\end{array}$ & $\begin{array}{c}\text { A 17-storey large panel residential } \\
\text { building, BS3-Tp Type (a 67-flat right- } \\
\text { end section with a 2-2-2-2 floor plan) }\end{array}$ \\
\hline 1.5 .5 & $\begin{array}{c}\text { A 17-storey large panel residential } \\
\text { building, BS4-Tp type (a 84-flat bend } \\
\text { section with a 1-1-1-3-3 floor plan) }\end{array}$ \\
\hline buildings ( $>16$ storeys) & $\begin{array}{c}\text { A 17-storey large panel residential } \\
\text { building, BS7-R type (a 84-flat bend } \\
\text { section with a 1-1-1-1-2-2 floor plan) }\end{array}$ \\
\hline
\end{tabular}

Table 2. General design information.

\begin{tabular}{|c|c|c|c|c|c|c|}
\hline $\begin{array}{c}\text { No (type, } \\
\text { section, } \\
\text { design no) }\end{array}$ & Region & $\begin{array}{c}\text { Gross floor } \\
\text { area, sq. m. }\end{array}$ & $\begin{array}{c}\text { Net floor } \\
\text { area, sq. m. }\end{array}$ & Designed in & $\begin{array}{c}\text { State } \\
\text { expertise } \\
\text { approval } \\
\text { issued in }\end{array}$ \\
\hline 1 & 2 & 3 & 4 & 5 & 6 \\
\hline 1.1 & \multicolumn{2}{|c|}{ Section 1. Low-rise residential buildings (manors) and townhouses } \\
\hline 1.2 .1 & $\begin{array}{c}\text { Volgograd } \\
\text { region }\end{array}$ & 223.50 & 832.20 & 2014 & 2014 \\
\hline 1.2 .2 & $\begin{array}{c}\text { Republic of } \\
\text { Bashkortostan }\end{array}$ & 1492.20 & 1143.36 & 2011 & 2012 \\
\hline 1.2 .3 & $\begin{array}{c}\text { Khabarovsk } \\
\text { region }\end{array}$ & 4260.00 & 2905.20 & 2013 & 2014 \\
\hline 1.3 .1 & Vologda region & 4894.60 & 3510.36 & 2014 & 2014 \\
\hline 1.3 .2 & $\begin{array}{c}\text { Kaliningrad } \\
\text { region }\end{array}$ & 8813.11 & $\mathrm{n} / \mathrm{a}$ & 2012 & 2012 \\
\hline 1.3 .3 & $\begin{array}{c}\text { Krasnodar } \\
\text { region }\end{array}$ & 6771.80 & 4923.00 & 2013 & 2014 \\
\hline 1.3 .4 & $\begin{array}{c}\text { Krasnodar } \\
\text { region }\end{array}$ & 6771.80 & 4923.00 & 2013 & 2014 \\
\hline 1.3 .5 & $\begin{array}{c}\text { Murmansk } \\
\text { region }\end{array}$ & 6720.00 & $\mathrm{n} / \mathrm{a}$ & 2012 & 2012 \\
\hline 1.4 .1 & $\begin{array}{c}\text { Kaliningrad } \\
\text { region }\end{array}$ & 6090.88 & $\mathrm{n} / \mathrm{a}$ & 2012 & 2012 \\
\hline 1.4 .2 & $\begin{array}{c}\text { Krasnoyarsk } \\
\text { region }\end{array}$ & 10753.21 & 7494.23 & 2014 & 2014 \\
\hline 1.5 .1 & & $\mathrm{n} / \mathrm{a}$ & $\mathrm{n} / \mathrm{a}$ & 2011 & $\mathrm{n} / \mathrm{a}$ \\
\hline 1.5 .2 & & $\mathrm{n} / \mathrm{a}$ & $\mathrm{n} / \mathrm{a}$ & 2011 & $\mathrm{n} / \mathrm{a}$ \\
\hline 1.5 .3 & $\mathrm{n} / \mathrm{n} / \mathrm{a}$ & $\mathrm{n} / \mathrm{a} / \mathrm{a}$ & 2011 & $\mathrm{n} / \mathrm{a}$ \\
\hline 1.5 .4 & & $\mathrm{n} / \mathrm{a} / \mathrm{a}$ & 2011 & $\mathrm{n} / \mathrm{a}$ \\
\hline 1.5 .5 & & & & 2011 & $\mathrm{n} / \mathrm{a}$ \\
\hline 1.5 .6 & & & & & & \\
\hline
\end{tabular}


Table 3. Estimated construction costs.

\begin{tabular}{|c|c|c|c|c|}
\hline $\begin{array}{l}\text { No (type, } \\
\text { section, } \\
\text { design } \\
\text { no) }\end{array}$ & $\begin{array}{l}\text { Year } \\
\text { of } \\
\text { commi } \\
\text { ssionin } \\
\quad \text { g }\end{array}$ & $\begin{array}{l}\text { Estimated } \\
\text { construction } \\
\text { costs at basic } \\
\text { prices in } \\
2001 \text {, in } \\
\text { thousand } \\
\text { roubles }\end{array}$ & $\begin{array}{c}\text { Construct } \\
\text { ion costs } \\
\text { at the } \\
\text { time of } \\
\text { expertise, } \\
\text { in } \\
\text { thousand } \\
\text { roubles }\end{array}$ & Owner of the rights \\
\hline 1 & 2 & 3 & 4 & 5 \\
\hline 1.1 & \multicolumn{4}{|c|}{ Section 1. Low-rise residential buildings (manors) and townhouses } \\
\hline 1.2 .1 & $\mathrm{n} / \mathrm{a}$ & 3729.39 & 26605.64 & $\begin{array}{l}\text { EPROM Ltd, TIN (tax identification } \\
\text { number) 3446012489 TRRC (tax } \\
\text { registration reason code) 344601001 } \\
19 / 2 \quad \text { Raboche-Krestyanskaya St, } \\
400074 \text {, Volgograd, tel. } 78-11-85\end{array}$ \\
\hline 1.2 .2 & $\mathrm{n} / \mathrm{a}$ & 5687.96 & 82270.41 & $\begin{array}{l}\text { State unitary enterprise Bashagroprom } \\
\text { proekt } 59 \text { Kommunisticheskaya St, Ufa, } \\
\text { Republic of Bashkortostan }\end{array}$ \\
\hline 1.2 .3 & $\mathrm{n} / \mathrm{a}$ & 24003.18 & 155365.09 & $\begin{array}{l}\text { Municipal unitary enterprise } \\
\text { Komsomolskgor proekt project institute } \\
\text { 41 Kirova St, 681000, Komsomolsk-on- } \\
\text { Amur, Khabarovsk region }\end{array}$ \\
\hline 1.3 .1 & 2014 & 13858.96 & 119699.71 & 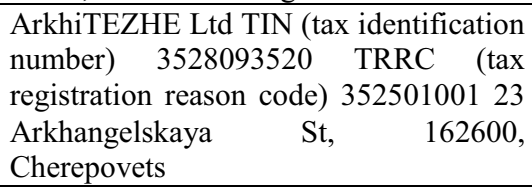 \\
\hline 1.3 .2 & $\mathrm{n} / \mathrm{a}$ & 29027.46 & 187526.39 & $\begin{array}{l}\text { Stroyproekt project institute Ltd, } 1 \\
\text { Kirova St, 236022, Kaliningrad }\end{array}$ \\
\hline 1.3 .3 & $\mathrm{n} / \mathrm{a}$ & 26453.57 & 81822.26 & $\begin{array}{l}\text { Terem Ltd, 128B Psekupskaya St, } \\
\text { 353290, Goryachy Klyuch, Krasnodar } \\
\text { region }\end{array}$ \\
\hline 1.3 .4 & $\mathrm{n} / \mathrm{a}$ & 26453.57 & 81822.26 & $\begin{array}{l}\text { Terem Ltd, 128B Psekupskaya St, } \\
\text { 353290, Goryachy Klyuch, Krasnodar } \\
\text { region }\end{array}$ \\
\hline 1.3 .5 & $\mathrm{n} / \mathrm{a}$ & 34824.12 & 219268.92 & $\begin{array}{l}\text { JSC Murmansk promproekt, } 43 \text { Lenina } \\
\text { St, } 183038 \text {, Murmansk }\end{array}$ \\
\hline 1.4 .1 & $\mathrm{n} / \mathrm{a}$ & 20030.59 & 132694.10 & $\begin{array}{l}\text { SIGMA-R Ltd, 3-1 Kolkhoznaya St, } \\
\text { 236009, Kaliningrad }\end{array}$ \\
\hline 1.4 .2 & $\mathrm{n} / \mathrm{a}$ & 55815.81 & 346821.99 & $\begin{array}{l}\text { JSC Krasnoyarskgrazhdan proekt town } \\
\text { planning institute, } 126 \text { imenigazety } \\
\text { Krasnoyarskiy Robochiy, } 660095 \text {, } \\
\text { Krasnoyarsk }\end{array}$ \\
\hline 1.5.1 & $\mathrm{n} / \mathrm{a}$ & 20566.57 & $\mathrm{n} / \mathrm{a}$ & $\begin{array}{l}\text { JSC Bureau of Architectural and } \\
\text { Construction Systems and New } \\
\text { Technologies named after A. Yakushev, } \\
5 \text { Mashinostroyeniya Pervaya St, } \\
\text { 109088, Moscow }\end{array}$ \\
\hline 1.5 .2 & $\mathrm{n} / \mathrm{a}$ & 18377.24 & $\mathrm{n} / \mathrm{a}$ & $\begin{array}{l}\text { JSC Bureau of Architectural and } \\
\text { Construction Systems and New } \\
\text { Technologies named after A. Yakushev, } \\
5 \text { Mashinostroyeniya Pervaya St, } \\
\text { 109088, Moscow }\end{array}$ \\
\hline
\end{tabular}




\begin{tabular}{|c|c|c|c|c|}
\hline 1.5 .3 & $\mathrm{n} / \mathrm{a}$ & 18904.63 & $\mathrm{n} / \mathrm{a}$ & $\begin{array}{l}\text { JSC Bureau of Architectural and } \\
\text { Construction Systems and New } \\
\text { Technologies named after A. Yakushev, } \\
5 \text { Mashinostroyeniya Pervaya St, } \\
\text { 109088, Moscow }\end{array}$ \\
\hline 1.5 .4 & $\mathrm{n} / \mathrm{a}$ & 18994.80 & $\mathrm{n} / \mathrm{a}$ & $\begin{array}{l}\text { JSC Bureau of Architectural and } \\
\text { Construction Systems and New } \\
\text { Technologies named after A. Yakushev, } \\
5 \text { Mashinostroyeniya Pervaya St, } \\
\text { 109088, Moscow }\end{array}$ \\
\hline 1.5 .5 & $\mathrm{n} / \mathrm{a}$ & 21681.24 & $\mathrm{n} / \mathrm{a}$ & $\begin{array}{l}\text { JSC Bureau of Architectural and } \\
\text { Construction Systems and New } \\
\text { Technologies named after A. Yakushev, } \\
5 \text { Mashinostroyeniya Pervaya St, } \\
\text { 109088, Moscow }\end{array}$ \\
\hline 1.5 .6 & $\mathrm{n} / \mathrm{a}$ & 20429.95 & $\mathrm{n} / \mathrm{a}$ & $\begin{array}{l}\text { JSC Bureau of Architectural and } \\
\text { Construction Systems and New } \\
\text { Technologies named after A. Yakushev, } \\
5 \text { Mashinostroyeniya Pervaya St, } \\
\text { 109088, Moscow }\end{array}$ \\
\hline
\end{tabular}

Table 4. Design specifications.

\begin{tabular}{|c|c|c|c|}
\hline $\begin{array}{l}\text { No (type, } \\
\text { section, } \\
\text { design no) }\end{array}$ & Portfolio link & $\begin{array}{l}\text { Ministry of } \\
\text { Construction } \\
\text { order no, } \\
\text { issued }\end{array}$ & Construction information \\
\hline 1 & 2 & 3 & 4 \\
\hline 1.1 & \multicolumn{3}{|c|}{ Section 1. Low-rise residential buildings (manors) and townhouses } \\
\hline 1.2 .1 & $\begin{array}{l}\text { http://www.min } \\
\text { stroyrf.ru/docs/1 } \\
0485 /\end{array}$ & $\begin{array}{c}\text { № 13/pr issued } \\
\text { on 19/01/2016 }\end{array}$ & $\begin{array}{l}\text { Basement: } 400-500 \mathrm{~mm} \text { foundation concrete } \\
\text { blocks. Exterior walls: } 9.3 \text { or } 10.7 \mathrm{NF} \\
\text { ceramic porous blocks, M100 grade concrete }\end{array}$ \\
\hline 1.2 .2 & $\begin{array}{c}\text { http://www.min } \\
\text { stroyrf.ru/docs/1 } \\
0486 /\end{array}$ & $\begin{array}{c}\text { № } 13 / \mathrm{pr} \\
\text { issued on } \\
19 / 01 / 2016 \\
\end{array}$ & $\begin{array}{l}\text { Exterior walls: silicate brick masonry with } \\
\text { heat-insulation and a thin layer of decorative } \\
\text { plastering }\end{array}$ \\
\hline 1.2 .3 & $\begin{array}{l}\text { http://www.min } \\
\text { stroyrf.ru/docs/1 } \\
0487 /\end{array}$ & $\begin{array}{l}\text { № } 13 / \mathrm{pr} \\
\text { issued on } \\
\text { 19/01/2016 }\end{array}$ & $\begin{array}{l}\text { Exterior walls: three-layered inner brick } \\
\text { masonry ( } 380 \mathrm{~mm} \text { heavy dense clay bricks), } \\
\text { a } 270 \mathrm{~mm} \text { layer of foam concrete } \\
\text { (insulation), a } 120 \mathrm{~mm} \text { layer of facing } \\
\text { masonry }\end{array}$ \\
\hline 1.3 .1 & $\begin{array}{c}\text { http://www.min } \\
\text { stroyrf.ru/docs/9 } \\
102 /\end{array}$ & $\begin{array}{l}\text { № } 483 / \mathrm{pr} \\
\text { issued on } \\
03 / 07 / 2015\end{array}$ & $\begin{array}{l}\text { Exterior walls: silicate brick masonry. } \\
\text { Floors and Ceilings: pre-fabricated } \\
\text { reinforced concrete panels. Tongue-and- } \\
\text { groove partition walls. Prefabricated } \\
\text { reinforced concrete stairs. Technoelast } \\
\text { welding roofing material. Windows: PVC } \\
\text { panels with double glazing. Insulated metal } \\
\text { entrance doors, metal front doors, wooden } \\
\text { interior doors }\end{array}$ \\
\hline 1.3 .2 & $\begin{array}{l}\text { http://www.min } \\
\text { stroyrf.ru/docs/1 } \\
0488 /\end{array}$ & $\begin{array}{l}\text { № 13/ pr } \\
\text { issued on } \\
\text { 19/01/2016 }\end{array}$ & $\begin{array}{l}\text { Exterior walls: pre-fabricated reinforced } \\
\text { concrete panels. Pre-fabricated reinforced } \\
\text { concrete floors and ceilings }\end{array}$ \\
\hline 1.3 .3 & $\begin{array}{c}\text { http://www.min } \\
\text { stroyrf.ru/docs/1 } \\
0489 /\end{array}$ & $\begin{array}{l}\text { № 13/ pr } \\
\text { issued on } \\
\text { 19/01/2016 }\end{array}$ & $\begin{array}{l}\text { Exterior walls: non-load-bearing two- } \\
\text { layered masonry ( } 120 \mathrm{~mm} \text { standard bricks })\end{array}$ \\
\hline
\end{tabular}




\begin{tabular}{|c|c|c|c|}
\hline 1.3 .4 & $\begin{array}{l}\text { http://www.min } \\
\text { stroyrf.ru/docs/1 } \\
0489 /\end{array}$ & $\begin{array}{l}\text { № } 13 / \mathrm{pr} \\
\text { issued on } \\
19 / 01 / 2016\end{array}$ & $\begin{array}{l}\text { Exterior walls: non-load-bearing, two- } \\
\text { layered masonry ( } 120 \mathrm{~mm} \text { standard bricks) }\end{array}$ \\
\hline 1.3 .5 & $\begin{array}{l}\text { http://www.min } \\
\text { stroyrf.ru/docs/1 } \\
0490 /\end{array}$ & $\begin{array}{c}\text { № 13/ pr } \\
\text { issued on } \\
19 / 01 / 2016\end{array}$ & $\begin{array}{l}\text { Exterior walls: concrete columns and beams } \\
\text { with brick walls and facing masonry }\end{array}$ \\
\hline 1.4 .1 & $\begin{array}{l}\text { http://www.min } \\
\text { stroyrf.ru/docs/1 } \\
0491 /\end{array}$ & $\begin{array}{c}\text { № 13/пр } \\
\text { issued on } \\
\text { 19/01/2016 }\end{array}$ & $\begin{array}{l}\text { Exterior walls: pre-fabricated reinforced } \\
\text { concrete panels. Pre-fabricated reinforced } \\
\text { concrete floors and ceilings }\end{array}$ \\
\hline 1.4 .2 & $\begin{array}{l}\text { http://www.min } \\
\text { stroyrf.ru/docs/1 } \\
0492 /\end{array}$ & $\begin{array}{l}\text { № } 13 / \mathrm{pr} \text { issued } \\
\text { on } 19 / 01 / 2016\end{array}$ & $\begin{array}{l}\text { Exterior walls: non-load-bearing masonry } \\
(250 \times 120 \times 65 \text { heavy dense bricks, KR-r } \\
\text { brand), a } 150 \mathrm{~mm} \text { layer of Technoroof } \\
\text { insulation material. The outer layer: } \\
\text { Kraspan ventilated facade system with } \\
\text { steel composite panels } \\
\begin{array}{l}\text { Floors and Ceilings: } 200 \mathrm{~mm} \text { thick } \\
\text { reinforced concrete panels }\end{array}\end{array}$ \\
\hline 1.5 .1 & $\begin{array}{l}\text { http://www.min } \\
\text { region.ru/upload } \\
\text { /documents } \\
\text { /reestr_1_7/reest } \\
\text { r_2/30102013_r } \\
\text { _5_1_1.pdf }\end{array}$ & $\begin{array}{l}\text { № } 457 \text { issued } \\
\text { on } 28 / 10 / 2013\end{array}$ & $\begin{array}{l}\text { The design of the section: longitudinal and } \\
\text { transverse large panel load-bearing walls } \\
\text { with exterior brick walls bearing on the floor } \\
\text { below. Interior walls: pre-fabricated } \\
\text { reinforced heavy weight concrete panels. } \\
\text { Floors and ceilings: solid reinforced heavy } \\
\text { weight concrete panels. Pre-fabricated } \\
\text { reinforced concrete balconies. The roof with } \\
\text { the heated attic floor and an interior drainage } \\
\text { system. }\end{array}$ \\
\hline 1.5 .2 & - & $\begin{array}{l}\text { № } 457 \text { issued } \\
\text { on } 28 / 10 / 2013\end{array}$ & $\begin{array}{l}\text { The design of the section: longitudinal and } \\
\text { transverse large panel load-bearing walls } \\
\text { with exterior brick walls bearing on the floor } \\
\text { below. Interior walls: pre-fabricated } \\
\text { reinforced heavy weight concrete panels. } \\
\text { Floors and ceilings: solid reinforced heavy } \\
\text { weight concrete panels. Pre-fabricated } \\
\text { reinforced concrete balconies. The roof with } \\
\text { the heated attic floor and an interior drainage } \\
\text { system. }\end{array}$ \\
\hline 1.5 .3 & $\begin{array}{l}\text { http://www.min } \\
\text { region.ru/upload } \\
\text { /documents } \\
\text { /reestr_1_7/reest } \\
\text { r_2/30102013_r } \\
\text { 5_1_2.pdf }\end{array}$ & $\begin{array}{l}\text { № } 457 \text { issued } \\
\text { on } 28 / 10 / 2013\end{array}$ & $\begin{array}{l}\text { The design of the section: longitudinal and } \\
\text { transverse large panel load-bearing walls } \\
\text { with exterior brick walls bearing on the floor } \\
\text { below. Interior walls: pre-fabricated } \\
\text { reinforced heavy weight concrete panels. } \\
\text { Floors and ceilings: solid reinforced heavy } \\
\text { weight concrete panels. Pre-fabricated } \\
\text { reinforced concrete balconies. The roof with } \\
\text { the heated attic floor and an interior drainage } \\
\text { system. }\end{array}$ \\
\hline 1.5 .4 & - & $\begin{array}{l}\text { № } 457 \text { issued } \\
\text { on } 28 / 10 / 2013\end{array}$ & $\begin{array}{l}\text { The design of the section: longitudinal and } \\
\text { transverse large panel load-bearing walls } \\
\text { with exterior brick walls bearing on the floor } \\
\text { below. Interior walls: pre-fabricated } \\
\text { reinforced heavy weight concrete panels. } \\
\text { Floors and ceilings: solid reinforced heavy } \\
\text { weight concrete panels. Pre-fabricated } \\
\text { reinforced concrete balconies. The roof with } \\
\text { the heated attic floor and an interior drainage } \\
\text { system. }\end{array}$ \\
\hline
\end{tabular}




\begin{tabular}{|c|c|c|c|}
\hline 1.5 .5 & $\begin{array}{l}\text { http://www.min } \\
\text { region.ru/upload } \\
\text { /documents/ } \\
\text { reestr_1_7/reest } \\
\text { r_2/30102013_r } \\
\text { _5_1_3.pdf }\end{array}$ & $\begin{array}{l}\text { № } 457 \text { issued } \\
\text { on } 28 / 10 / 2013\end{array}$ & $\begin{array}{l}\text { The design of the section: longitudinal and } \\
\text { transverse large panel load-bearing walls } \\
\text { with exterior brick walls bearing on the floor } \\
\text { below. Interior walls: pre-fabricated } \\
\text { reinforced heavy weight concrete panels. } \\
\text { Floors and ceilings: solid reinforced heavy } \\
\text { weight concrete panels. Pre-fabricated } \\
\text { reinforced concrete balconies. The roof with } \\
\text { the heated attic floor and an interior drainage } \\
\text { system. }\end{array}$ \\
\hline 1.5 .6 & - & $\begin{array}{l}\text { № } 457 \text { issued } \\
\text { on } 28 / 10 / 2013\end{array}$ & $\begin{array}{l}\text { The design of the section: longitudinal and } \\
\text { transverse large panel load-bearing walls } \\
\text { with exterior brick walls bearing on the floor } \\
\text { below. Interior walls: pre-fabricated } \\
\text { reinforced heavy weight concrete panels. } \\
\text { Floors and ceilings: solid reinforced heavy } \\
\text { weight concrete panels. Pre-fabricated } \\
\text { reinforced concrete balconies. The roof with } \\
\text { the heated attic floor and an interior drainage } \\
\text { system. }\end{array}$ \\
\hline
\end{tabular}

In order to analyze the data for each design, all construction costs need to be adjusted to a single price level. This article uses the adjustment indices of estimated construction costs issued by the Ministry of Construction of the Russian Federation [3].

Table 5. Estimated construction costs adjusted to 2016 prices.

\begin{tabular}{|c|c|c|c|c|c|}
\hline No & Design & $\begin{array}{l}\text { Price of } 1 \\
\text { sq. } \mathrm{m} \text {. in } \\
2001 \text {, in } \\
\text { roubles }\end{array}$ & $\begin{array}{l}\text { Adjustment } \\
\text { index }\end{array}$ & $\begin{array}{l}\text { Price of } 1 \\
\text { sq. } m \text { in } \\
2016 \text {, in } \\
\text { roubles }\end{array}$ & $\begin{array}{c}\text { Average market } \\
\text { price of } 1 \text { sq. } m \text { of } \\
\text { housing } \\
\text { accommodation in } \\
\text { the regions of the } \\
\text { Russian } \\
\text { Federation in the } \\
\text { 1st quarter of } \\
2016 \text {, in roubles } \\
\text { [5,6] }\end{array}$ \\
\hline 1 & 2 & 3 & 4 & 5 & 6 \\
\hline 1 & $\begin{array}{l}\text { A 24-flat residential } \\
\text { building for orphans } \\
\text { in the town of } \\
\text { Nikolaevsk, } \\
\text { Volgograd region } \\
\end{array}$ & 3048 & 8.551 & 26065 & 31100.00 \\
\hline 2 & $\begin{array}{l}\text { A residential building } \\
\text { at } 15 \mathrm{a} \text { Montclair St, } \\
112 \text { th district of the } \\
\text { city of Cherepovets, } \\
\text { Vologda region }\end{array}$ & 2831 & 9.596 & 27171 & 35375.00 \\
\hline 3 & $\begin{array}{l}\text { A 108-flat residential } \\
\text { building in Levitana } \\
\text { St, Kaliningrad region }\end{array}$ & 3294 & 11.888 & 39155 & 32915.00 \\
\hline 4 & $\begin{array}{l}\text { An } 88 \text {-flat residential } \\
\text { building, Kaliningrad } \\
\text { region }\end{array}$ & 3289 & 11.888 & 39095 & 32915.00 \\
\hline
\end{tabular}




\begin{tabular}{|c|c|c|c|c|c|}
\hline 5 & $\begin{array}{l}\text { An apartment building } \\
\text { at no1 Vishnevaya St, } \\
\text { in the Central district } \\
\text { of Sochi }\end{array}$ & 3906 & 9.308 & 36361 & 34835.00 \\
\hline 6 & $\begin{array}{l}\text { An apartment building } \\
\text { at no } 2 \text { Vishnevaya St, } \\
\text { in the Central district } \\
\text { of Sochi }\end{array}$ & 3906 & 9.308 & 36361 & 34835.00 \\
\hline 7 & $\begin{array}{l}\text { A residential building } \\
\text { for the artistic } \\
\text { personnel of regional } \\
\text { theatres and other } \\
\text { entertainment } \\
\text { establishments in the } \\
\text { city of Krasnoyarsk }\end{array}$ & 5191 & 9.872 & 51242 & 39238.00 \\
\hline 8 & $\begin{array}{l}\text { A residential building } \\
\text { in Kirpichnaya St, in } \\
\text { the city of Murmansk }\end{array}$ & 5182 & 13.623 & 70597 & 37202.00 \\
\hline 9 & $\begin{array}{l}\text { A 24-flat residential } \\
\text { building in Iglino, } \\
\text { Iglinsky } \\
\text { administrative } \\
\text { municipal and } \\
\text { Republic district, } \\
\text { Bashkortostan }\end{array}$ & 3812 & 9.076 & 34596 & 34586.00 \\
\hline 10 & $\begin{array}{l}\text { A residential building } \\
\text { for the disabled, } \\
\text { orphans and families } \\
\text { with disabled children } \\
\text { in the city of } \\
\text { Khabarovsk }\end{array}$ & 5635 & 12.429 & 70032 & 46018.00 \\
\hline
\end{tabular}

The analysis of the adjusted construction costs raises questions about whether it is appropriate to re-use these designs. Seven designs out of ten exceed the average market prices of housing accommodation. Note that we intentionally chose to analyze residential buildings, as the market for housing accommodation is open, active and does not allow for much correction in terms of construction costs estimation and operation requirements [7].

The compilation of the Registry of Standardized Design has the aim of reducing budget expenditures. However, the share of government budget that goes to residential construction is minimal. The main budget spendings are associated with the construction of public facilities, i.e. schools, nurseries, sports venues [4]. The analysis of the data currently published in the Registry of Standardized Designs revealed numerous in consistencies where the designs added to the Registry fail to comply with the requirements for designs approved for repeat use as stipulated in the legislation of the Russian Federation.

\section{Conclusion}

It can be conclusion that now there exist numerous issues regarding the contents of the Registry and the viability of the designs added to it. Below is the summary of the issues:

- ambiguity of the criteria for the selection of a design by the investor (developer);

- poor quality of the contents that stems from insufficient requirements for the portfolio of standardized designs;

- lack of legislation regulating repeat design siting, expertise and operation; 
- ambiguity in terms of respecting intellectual property rights for the repeat design documentation.

Yet, standardized designs remain a powerful tool the principal benefits of which are time and cost savings. We suggest that new information be added to the Registry to reflect the following: maintenance and operations experience; design drawbacks; procedures for routine and major repairs for specific designs, construction and finishing materials. Local features, such as climate, material resources and workforce market, also need to be taken into consideration.

\section{References}

1. Standardized Designs, URL: http://gostbank.metaltorg.ru/data/norms_new/snip/76.pdf

2. Notes on Standardized Designs, URL: http://www.ge-mchs.ru/tproekt

3. O. Didkovskaya, SSUACU, 201-208 (2015)

4. President's Message to the Federal Assembly, http://www.consultant.ru/document/cons_doc_law_155646/

5. Official Statistics, http://www.gks.ru/free doc/new site/business/stroit/stroi_graf.htm

6. http://www.garant.ru/products/ipo/prime/doc/56556842/\#ixzz40JMWmHVt

7. O. Didkovskaya, Fundamental Research 11(5), 986-993 (2015)

8. The Registry of Standardized Designs, http://www.minstroyrf.ru/docs/1482/

9. M. Kisternaya, V. Kozlov, I. Grishina, M. Leri, Dendrochronologia 40, 143-150 (2016)

10. J. M. Beaver, Y. Lai, S. J. Rolle, Y. Liu, DNA Repair 48, 17-29 (2016)

11. F. Ceschin, I. Gaziulusoy, Design Studies 47, 118-163 (2016) 